\title{
The place of comorbidity and of subtle psychopathological evaluation
}

\author{
Hans-Jürgen Möller ${ }^{1}$ \\ Published online: 23 October 2020 \\ (c) Springer-Verlag GmbH Germany, part of Springer Nature 2020
}

While in the traditional disorder-related classification systems and in the early versions of DSM and ICD an individual diagnosis referred to only one disease or disorder and other potential concurrent diagnoses were not permitted because of hierarchical rules, the modern versions of ICD and DSM allow comorbidity in the sense, that a person can have e.g. a major depressive disorder and an obsessive- compulsive disorder [1]. The introduction of the concept of comorbidity, partially a consequence of the restrictive operationalisation of the different mental disorders, was understood as important step forward in the description of mental disorders. These kinds of comorbidities are quite common. While in the traditional psychopathology these additional symptoms, now diagnosed as comorbidity, were seen as part of the respective disorder, as a special enrichment of the core pathognomonic clinical picture, or as pre-runner of the fullblown illness (e.g. compulsive symptoms in the predromal state of schizophrenia, e.g. anxiety symptoms as precursor of MDD), the concept of comorbidity regards them as a disorder per se and thus opens a plethora of opportunities for specifically related research activities.

This can be seen in the current literature where results on comorbidity and co-symptomatology seem sometimes to predominate studies on disorders without comorbid conditions [2-7]. However, depending on the design of the studies, it is not always clear whether e.g. the neurobiological research result is really related to the comorbid condition or to the first diagnosis. Additionally, the question, whether the result, e.g. a neurobiological finding, for the comorbid diagnosis is congruent with respective results for the same diagnosis/disorder in a non-comorbid condition, often cannot be answered. Does all this lead to a better/additional understanding of the neurobiological underpinnings of each of the respective disorders, to more than can be reached by

Hans-Jürgen Möller

Hans-Juergen.Moeller@med.uni-muenchen.de

1 Department of Psychiatry and Psychotherapy, University Hospital, LMU Munich, München, Germany studying each of the respective disorders separately? Do we learn something more about the neurobiological background of the interaction of two comorbid disorders? Are these questions, which should be considered in all the studies on comorbidity? Does this finally lead to a new perspective beyond the frame of categorical or dimensional diagnostic approaches to these disorders? Will finally new concepts of psychopathology or of disorders be a result of all these research activities?

Are there other ways to address the issue of comorbidity without using the concepts of given disorders and without throwing away the primary symptoms based approach, as done by the RDoC, which focuses primarily on a neurobiological conceptualization. Unlike this neurobiological approach of the RDoC, which cannot directly be translated in clinical concepts, the psychopathological approach is considered to be a suitable basis for a symptom oriented classification system that is built on modern methods of quantitative classification. According to a large number of empirical studies, psychopathology tend to be rather dimensional than categorical and research supports the hypothesis that symptoms are on a continuum rather than being discrete. Studies have also shown how psychopathological dimensions can be arranged hierarchically, ranging from a "broad spectrum level" dimension to specific and narrow clusters of symptoms. In this way a quantitative approach could solve the "problem of comorbidity" by explicitly modelling patterns of co-occurring signs and symptoms within a detailed and variegated hierarchy of dimensional concepts, with direct clinical usefulness. This interesting approach seems currently being realised by the "Hierarchical Taxonomy of Psychopathology (HiTOP) Consortium" [8]. But this approach is widely criticized for various reasons. Besides methodological issues, such as the required quality of the rating scales and the persistency of cross-sectional findings in the long-term, criticism was expressed about the translation into clinical settings. As especially problematic were seen the model's lack of course- and cause-related aspects [9]. Nevertheless, this interesting methodological approach should be further explored. 
Going back to the categorical and dimensional concepts used in DSM-5 and ICD-10 the definitions/operationalisations of the respective disorders might be too simple/reductionistic and/ or in clinical and even research practice the psychopathological explorations/differentiations might not be sufficiently subtle/sophisticated, which can induce problematic diagnostic pitfalls. This is the message of two interesting studies reported in this issue, both are from the research group around Parnas, known for its interest in self disorders as indicator of schizophrenia spectrum disorders [10]. Zandersen and Parnas in a very careful and timeconsuming sophisticated psychiatric evaluation, involving several senior level clinical psychiatrists and researchers and a comprehensive battery of psychopathological scales, found that a high proportion of patients diagnosed in Danish outpatient settings as borderline personality disorders (BPD) had to be re-diagnosed. 67\% in DSM-5 and 77\% in ICD-10 in fact met the criteria for a schizophrenia spectrum disorder, i.e. schizophrenia or schizotypal personality disorder. The schizophrenic spectrum group scored significantly higher on the level of disorders of core self as measured with the Examination of Anomalous Self -Experiences Scale (EASE). The BPD criterion of "identity disturbance" was significantly correlated with the mean total score of EASE. These findings are discussed in the light of changes from prototypical to polythetic diagnostic systems. From the same research group around Parnas another interesting paper (Rasmusssen, Nordgaard and Parnas) in the current issue of EAPCN describes the value of a careful diagnostic procedure to explore schizophrenia spectrum psychopathology in obsessive-compulsive disorder (OCD). The study examined in a sophisticated approach, like in the study mentioned above, the lifetime schizophrenia spectrum psychopathology, including subtle schizotypal symptomatology and subjective anomalies such as self-disorders and also the differentiation between true obsessions and pseudo-obsessions in a sample diagnosed with OCD in two Danish specialized settings. The patients underwent semi-structured, narrative interviews assessing a comprehensive test-battery of psychopathological instruments. The final lifetime diagnosis was based on a consensus between a senior clinical psychiatrist and an experienced research clinician. The study found that $29 \%$ of the patients fulfilled criteria of schizophrenia or another non-affective psychosis as main lifetime research diagnosis. Another 33\% received a research-diagnosis of schizotypal personality disorder. Only $29 \%$ got the main diagnosis of
OCD. The conclusion of the paper is that a high proportion of clinically diagnosed OCD patients fulfil diagnostic criteria of a schizophrenic spectrum disorder.

The general conclusion of both papers is apparently that a more sophisticated differentiation of psychopathological phenomena like disorders of core self vs. identity disturbances, true obsessions vs. pseudo-obsessions etc.is necessary to avoid diagnostic pitfalls. The question arises how neurobiological research on mental disorders can proceed successfully if we don`t take care sufficiently for a sophisticated diagnostic approach beyond the diagnostic criteria of DSM-5 and ICD-10/11.

\section{References}

1. Moeller HJ (2020) Classification systems of mental disorders: where did we go wrong? In: Pompili M, McIntyre R, Fiorillo A, Sartorius N (eds) New directions in psychiatry. Springer, Berlin, pp 193-2004

2. Angst J, Merikangas KR, Cui L et al (2018) Bipolar spectrum in major depressive disorders. Eur Arch Psychiatry Clin Neurosci 268:741-748

3. Menghini D, Armando M, Calcagni M et al (2018) The influence of Generalized Anxiety Disorder on Executive Functions in children with ADHD. Eur Arch Psychiatry Clin Neurosci 268:349-357

4. Preuss UW, Gouzoulis-Mayfrank E, Havemann-Reinecke U et al (2018) Psychiatric comorbidity in alcohol use disorders: results from the German S3 guidelines. Eur Arch Psychiatry Clin Neurosci 268:219-229

5. Schirmbeck F, Kiss A, Eberhardt K et al (2018) Obsessive-compulsive symptoms and overall psychopathology in psychotic disorder: longitudinal assessment of patients and siblings. Eur Arch Psychiatry Clin Neursosci 268:279-289

6. Swets M, Schirmbeck F, Dekker J et al (2019) Longitudinal association between motor and compulsive symptoms in patients with psychosis and their unaffected siblings. Eur Arch Psychiatry Clin Neurosci 269:257-268

7. Himmerich H, Hotopf M, Shetty H et al (2019) Psychiatric comorbidity as a risk factor for mortality in people with anorexia nervosa. Eur Arch Pschiatry Clin Neurosci 269:351-359

8. Krueger RR, Kotov R, Watson D et al (2018) Progress in achieving quantitative classification of psychopathology. World Psychiatry 17:282-293

9. Wittchen HU, Beesdo-Baum K (2018) "Throwing out the baby with the bathwater"? Conceptual and methodological limitations of the HiTOP approach. World Psychiatry 17:280-299

10. Nordgaard J, Nilsson LS, Siersbaek N, Saebye D et al (2018) Self disorders in schizophrenia spectrum disorders: a five year followup study. Eur Arch Psychiatry Clin Neurosci 268:713-718 